\title{
Ivacaftor in cystic fibrosis: the first disease modifying agent
}

\author{
Suruchi Aditya
}

Department of Pharmacology, Dr. Harvansh Singh Judge Institute of Dental Sciences, Panjab University, Chandigarh, India

Received: 30 September 2012 Accepted: 13 October 2012

\author{
Correspondence to: \\ Dr. Suruchi Aditya, \\ Email: \\ suruchiaditya@rediffmail.com
}

\begin{abstract}
The exact magnitude of cystic fibrosis (CF) in India is not known, as it is often misrepresented and underdiagnosed. $\mathrm{CF}$ is caused by a mutation in the gene that encodes for the CF transmembrane conductance regulator (CFTR) protein whose dysfunction leads to multiorgan manifestations. Most $\mathrm{CF}$ mutations either reduce the number of CFTR channels at the cell surface or impair the channel function. Current treatments (mucolytics, antibiotics and antiinflammatory agents) target the secondary effects of CFTR dysfunction and help to ameliorate the symptoms but do not address the basic defect of the disease. Ivacaftor is a first-in-class oral CFTR potentiator that increases the CFTR channel opening. In clinical trials, ivacaftor has shown improved pulmonary function, normalization of sweat chloride concentration, substantial weight gain as well as acceptable safety profile. The most frequent adverse effects associated with ivacaftor include headache, oropharyngeal pain, upper respiratory tract infection, nasal congestion, abdominal pain and nasopharyngitis. FDA has approved this agent for the treatment of $\mathrm{CF}$ in patients aged 6 years or older with at least one copy of the G551D mutation in the CFTR gene. Searches of medline, cochrane database, medscape, SCOPUS and clinicaltrials.org were made for terms like CFTR potentiator, cystic fibrosis, and ivacaftor. Relevant journal articles from last 5 years were chosen.
\end{abstract}

Keywords: Cystic fibrosis transmembrane conductance regulator (CFTR), Ivacaftor, CFTR potentiator

\section{INTRODUCTION}

Once thought to be an extremely rare disease in the Indian subcontinent, availability of newer improved genetic and biochemical testing point towards a probable increased incidence of cystic fibrosis (CF). A lethal autosomal recessive genetic disease, $\mathrm{CF}$ is most common in Caucasians and the prevalence varies with the ethnic origin of the population. There is evidence of extensive allelic heterogeneity in Indian patients as compared to Mediterranean and European population owing to diverse ethnic origin. ${ }^{1-3}$

Patients with $\mathrm{CF}$ show a complex spectrum of disease phenotypes. Clinically, classical CF is characterized by faulty chloride transport leading to accumulation of dehydrated and hyper viscous mucus that compromises mucociliary clearance and makes airways more vulnerable to infection (particularly with Pseudomonas aeruginosa) and inflammation, ultimately leading to airway destruction, respiratory failure and death. ${ }^{4}$ There are protracted periods of clinical stability erupted by pulmonary exacerbations, often triggered by a viral infection and defined by increased cough, weight loss, low grade fever, increased sputum volume, and decrements in pulmonary function. Deregulated sodium transport is an additional phenomenon that plays a role in pathophysiology of $\mathrm{CF}$ lung disease. ${ }^{5}$ CFTR (CF transmembrane conductance regulator) dysfunction leads to multiorgan manifestations including pancreatic insufficiency, hepatic dysfunction, intestinal malabsorption and reproductive abnormalities. Sweat with elevated chloride $(>60 \mathrm{mmol} / \mathrm{L})$ is nearly pathognomonic. $\mathrm{CF}$ related disorders include congenital absence of vas deferens, isolated idiopathic pancreatitis, chronic rhinosinusitis, nasal polyposis and idiopathic bronchiectasis.

$\mathrm{CF}$ is caused by genetic mutations in the CFTR gene, located on the long arm of chromosome 7 (7q31.2) that undergoes transcription and is translated into CFTR protein that moves to the cell membrane, where it mainly 
functions as the predominant chloride channel. ${ }^{4}$ The CFTR protein is a single polypeptide chain, containing 1480 amino acids. Over 1900 mutations have been described in the CFTR gene, which are broadly categorized into six classes involving truncation, processing, activation, channel conductance, splice mutations and protein instability. ${ }^{6,7}$ While class IV and V are milder forms, severe phenotypes are seen in classes I, II, III and VI.

Class I mutations $(\sim 10 \%)$ such as G542X result from premature termination codons (PTC) that cause premature truncation of normal protein translation resulting in an inability of the channel to reach the cell membrane.

Class II mutations result from trafficking defects that cause misfolding of CFTR protein so that it is not transported to cell surface; it remains in endoplasmic reticulum and is degraded by proteasome. However, little residual CFTR is maintained as seen in $\triangle \mathrm{F} 508$, the most common mutation in humans $(66-70 \%)$ worldwide, where a deletion $(\Delta)$ of three nucleotides results in a loss of the amino acid phenylalanine $(F)$ at the 508th position on the protein leading to a partially functional anion channel.

Class III mutations ( 2-3\%) result from gating mutations that are characterized by full length CFTR reaching the cell surface but exhibiting reduced ion transport activity owing to abnormal channel gating as seen in G551DCFTR, where glycine is substituted with aspartic acid at amino acid 551. It represents an archetype CFTR allele as it exhibits normal surface expression and half- life, but confers a severe defect in channel gating owing to amino acid substitution in adenosine triphosphate (ATP) binding pocket within the nucleotide binding domain. The channel does not open properly leading to impaired chloride transport.

In class IV $(<2 \%)$ mutations such as R117H, CFTR protein reaches cell membrane and some of the protein is functional. However channel narrowing hampers chloride transport.

Class V (splicing) mutations are least common and result in improper processing of mRNA leading to reduced number of CFTR proteins that reach the surface, but they are able to transport chloride effectively.

Class VI mutations result in impaired conductance of ions other than chloride. Defect in protein stability lead to reduced membrane residence time due to reduced half-life of complex-glycosylated truncated CFTR.

The life expectancy of CF patients is reduced (median survival is 37.4 years), and the treatment burden to maintain health is high, indicating a need for better therapies. Current therapeutic options treat downstream disease process as a result of defective CFTR without addressing the underlying genetic defect. Inhaled tobramycin, recombinant human deoxyribose nuclease (dornase alfa), azithromycin and hypertonic saline are used to improve lung function and reduce pulmonary exacerbations. ${ }^{7}$ Therapeutic strategies aimed at rescuing the abnormal protein that is either synthesized in reduced amounts or has poor anion conductance has lead to development of novel drugs. CFTR modulators are pharmacological agents intended to repair the CFTR protein. A compound that enhances CFTR trafficking to the membrane is termed a "corrector" (for mutation classes I and II) while an agent that increases the flow of ions through activated CFTR channels is named as a "potentiator" (for mutation classes III-VI).

There is now a promising pipeline of disease modifying agents currently under evaluation. Ataluren, allows read through of premature stop codons and is in Phase III clinical trials for treatment of truncation mutations (class I mutation defect). VX 809 (lumacaftor) is a corrector that modulates CFTR folding and trafficking so that the channel can reach the cell surface (class II mutation defect). Ivacaftor (VX 770) is a first- in-class drug for treatment of $\mathrm{CF}$ in patients aged 6 years or older who have a G551D mutation in the CFTR gene (class III mutation defect). ${ }^{8}$

Ivacaftor [N-(2, 4-di-tert-butyl-5-hydroxyphenyl)-4-oxo1, 4-dihydroquinone-3- carboxamide)] has molecular weight of 392.49 and molecular formula of $\mathrm{C}_{24} \mathrm{H}_{28} \mathrm{~N}_{2} \mathrm{O}_{3}$. The FDA approved dose of ivacaftor for patients aged at least 6 years with no hepatic or renal impairment is 150 mg taken orally every 12 hours.

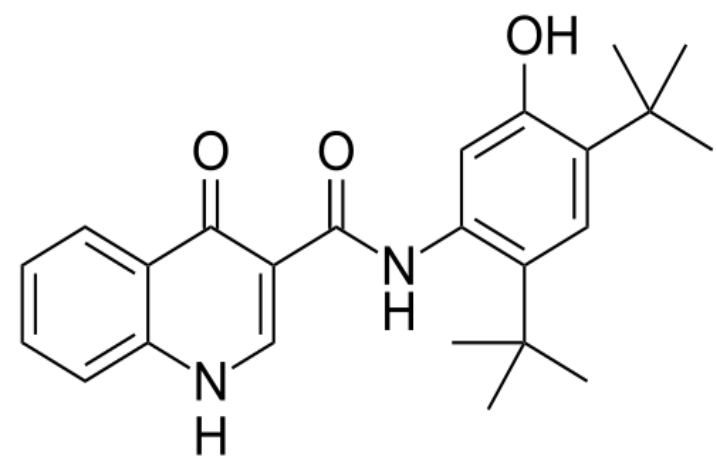

Figure 1: Molecular structure of ivacaftor.

\section{PHARMACOKINETICS}

Following oral administration, the systemic exposure of ivacaftor is increased approximately two to four fold when given with food containing fat (hence recommended along with eggs, butter, peanut butter, cheese pizza). Being extensively metabolized via cytochrome P450 (CYP), it has the potential to interact with inhibitors and inducers of CYP3A. One of the 
metabolites $\left(\mathrm{M}_{1}\right)$ has $1 / 6^{\text {th }}$ potency of parent compound, the other metabolite $\left(\mathrm{M}_{6}\right)$ is pharmacologically inactive. $90 \%$ of the drug is eliminated in feces. Dosage should be reduced in moderate or severe hepatic impairment (ChildPugh class B and C). Caution should be exercised in patients with severe renal impairment or end stage renal disease. $^{9}$

\section{MECHANISM OF ACTION}

In G551D, mutations permit CFTR protein to reach the epithelial-cell surface, but the protein is defective in chloride transport. Ivacaftor is a CFTR potentiator designed to increase the duration that activated channels remain open at the cell surface for transporting chloride ions. It binds to an allosteric site distinct from catalytic site on CFTR and induces channel opening via a nonconventional mechanism which does not require ATP binding and hydrolysis. ${ }^{10}$

In vitro studies of air-liquid interface cultures of bronchial epithelial cells from the lungs of patients with CF have shown that correction of abnormal CFTR-mediated ion transport increases the air-surface fluid level and ciliary beat frequency leading to improvement in lung function. ${ }^{11}$

\section{CLINICAL TRIALS}

In a Phase II study conducted for four weeks, ivacaftor was well tolerated. Parameters such as nasal potential difference (NPD) (a measure of CFTR function), sweat chloride, forced expiratory volume in 1 second $\left(\mathrm{FEV}_{1}\right)$ showed statistically significant changes from baseline but not when compared to placebo. The Cystic Fibrosis Questionnaire-Revised (CFQR), a measurement tool to determine changes in health related quality of life showed clinically relevant improvements in the respiratory domain of the CFQ-R ( $\geq 4$ points) at the conclusion of ivacaftor treatment in part 2 of the study, but the improvements did not reach statistical significance $(8.3$ points versus 2.8 points; $\mathrm{p}=0.75) .^{12}$

Table 1: Phase II and III studies done with ivacaftor in cystic fibrosis patients with at least one copy of G551DCFTR mutation.

\begin{tabular}{|c|c|c|c|c|c|}
\hline Trial & Drug & $\begin{array}{l}\text { Absolute } \\
\text { change in } \mathrm{FEV}_{1} \\
\text { from baseline }\end{array}$ & $\begin{array}{l}\text { Mean change in } \\
\text { sweat chloride } \\
\text { from baseline } \\
(\mathrm{mEq} / \mathrm{L})\end{array}$ & $\begin{array}{l}\text { Weight change } \\
\text { from baseline } \\
\text { (kg) }\end{array}$ & $\begin{array}{l}\text { Other clinically } \\
\text { relevant observation } \\
\text { (NPD/CFQ-R) }\end{array}$ \\
\hline \multirow{2}{*}{$\begin{array}{l}\text { Accurso et } \\
\mathrm{al}^{12}, \text { Phase II } \\
(4 \text { weeks })\end{array}$} & Iva & $8.7 \%(\mathrm{p}=0.008)$ & $-59.5(\mathrm{p}=0.008)$ & - & $\begin{array}{l}\text { NPD }(\mathrm{mV}) \\
-3.5(\mathrm{p}=0.02)\end{array}$ \\
\hline & Placebo & $7.3 \%(\mathrm{p}=0.13)$ & $5.0(\mathrm{p}=0.88)$ & & $-0.4(\mathrm{p}=0.88)$ \\
\hline \multirow{2}{*}{$\begin{array}{l}\text { Ramsey et a } 1^{13} \\
\text { STRIVE study, } \\
\text { Phase III } \\
\text { (48 weeks) }\end{array}$} & Iva & $\begin{array}{l}10.1 \% \\
(p<0.0001)\end{array}$ & $-48.7 \quad(p<0.0001)$ & $\begin{array}{l}3.1 \\
(p<0.0001)\end{array}$ & $\begin{array}{l}\text { CFQ-R (median change } \\
\text { from baseline) } \\
5.9 \text { points }\end{array}$ \\
\hline & Placebo & $-\quad 0.4 \%$ & $\begin{array}{l}-\quad 0.8 \\
\end{array}$ & 0.4 & - 2.7 points \\
\hline \multirow{2}{*}{$\begin{array}{l}\text { Aherns et al }{ }^{14} \\
\text { Phase III } \\
\text { ENVISION study } \\
\text { (48 weeks) }\end{array}$} & Iva & $10 \%(\mathrm{p}=0.0006)$ & $\begin{array}{l}-55.53 \\
(p<0.0001)\end{array}$ & $\begin{array}{l}2.8 \\
(\mathrm{p}=0.0002) \\
\text { (versus placebo) }\end{array}$ & $\begin{array}{l}\text { CFQ-R } \\
6.31 \text { points }(p=0.109)\end{array}$ \\
\hline & Placebo & & 1.21 & & 0.25 points \\
\hline $\begin{array}{l}\text { McKone et al } \\
\text { Phase III } \\
\text { STRIVE } \\
\text { Extension, open } \\
\text { label study } \\
\text { (60 weeks) }\end{array}$ & & $\begin{array}{l}11.1 \pm 9.7 \% \\
\text { Relative change } \\
\text { of } \mathrm{FEV}_{1} \text { from } \\
\text { baseline }= \\
18.7 \% \pm 17 \%\end{array}$ & - & - & - \\
\hline
\end{tabular}

$\mathrm{NPD}=$ nasal potential difference, CFQ-R= Cystic Fibrosis Questionnaire-Revised, FEV1= Forced expiratory volume in 1 second, Iva= ivacaftor (150mg twice daily). 
In the STRIVE (study of ivacaftor in cystic fibrosis subjects aged 12 years and older with the G551D mutation) study, the addition of ivacaftor to established first-line treatment modalities (inhaled tobramycin, oral azithromycin, dornase alfa) resulted in statistically significant improvements in pulmonary function (a relative improvement of $17.2 \%$ in $\mathrm{FEV}_{1}$ over baseline), sweat chloride concentration and CFQ-R respiratory domain score (Table 1). At week 48, a total of $67 \%$ of subjects in the ivacaftor group were free from pulmonary exacerbations, as compared to $41 \%$ in the placebo group corresponding to a $55 \%$ risk reduction. ${ }^{13}$ The phase III ENVISION (study of ivacaftor in cystic fibrosis subjects aged 6 to 11 years with the G551D mutation) study showed statistically significant improvements in pulmonary function, sweat chloride and weight gain but not in the CFQ-respiratory domain score (Table 1). ${ }^{14}$

PERSIST (study of VX770 in cystic fibrosis subjects) is an ongoing Phase III, open-label, 96-week, rollover extension trial designed to further evaluate the long-term safety and efficacy of treatment with ivacaftor in patients who have completed 48 weeks of treatment in the Phase III STRIVE and ENVISION studies. STRIVE Extension, an open label study for an additional12 weeks after the STRIVE study, showed that addition of ivacaftor to established first-line treatment modalities resulted in significant improvements in pulmonary function and weight gain. $43.5 \%$ patients were free from pulmonary exacerbations at week 60 , confirming the long term effectiveness of ivacaftor (Table 1). ${ }^{15}$

The use of ivacaftor in $\Delta \mathrm{F} 508$ homozygous patients failed to increase the CFTR function, likely because there is a lack of CFTR channel available at the cell membrane. ${ }^{16}$ This has prompted evaluation of ivacaftor in conjunction with VX-809 (corrector) for treating this population. ${ }^{16}$

\section{ADVERSE EFFECTS}

Commonly observed adverse effects associated with ivacaftor include headache, oropharyngeal pain, upper respiratory tract infection, nasopharyngitis, diarrhea, nausea and dizziness. ${ }^{8,9}$ Because of the potential risk for hepatotoxicity, it is recommended to check alanine aminotransferase (ALT) and aspartate aminotransferase (AST) levels at baseline followed by reassessment every 3 months in first year of treatment and annually thereafter. It is a pregnancy category B medication. Concomitant use of a strong inducer or inhibitor of CYP $3 \mathrm{~A}$ should be avoided.

\section{FUTURE PERSPECTIVES}

An ideal treatment in CF should be safe for long periods of time with a convenient mode of administration particularly improving and protecting the organ system that gives rise to most of the morbidity and mortality (the lungs). Additionally, it should be suitable for use in infants in order to prevent lung damage entirely.
Introduction of ivacaftor represents a paradigm shift in treatment of $\mathrm{CF}$ from symptomatic treatment to a disease modifying drug that targets the underlying genetic defect. It represents the beginning of an era of genetic testing (genotyping) and imparting individualized therapy in CF. ${ }^{17}$

Although the structural lung damage may not be reversed, corrections of chloride transport defect may allow aggressive symptomatic therapy to become more effective and thereby reduce the manifestations of both pulmonary and extrapulmonary disease as well as the treatment burden. Initiation of therapy in infancy will not reverse pre-existing defect (such as congenital bilateral absence of the vas deferens, meconium ileus and pancreatic insufficiency), but progression of the disease might be halted.

In vitro studies have shown potential clinical benefit of ivacaftor in CF patients who have CFTR gating mutations beyond G551D, suggesting that ivacaftor is not a mutation specific CFTR potentiator. ${ }^{18}$ Combination of a corrector and potentiator may produce more benefit than a single agent alone.

\section{UNRESOLVED ISSUES}

A large number of $(>1500)$ relatively uncommon $(<2 \%$ each) mutations in CFTR gene makes genetic testing a challenging proposition. Identifying the specific mutation in a patient with subsequent selection of the appropriate therapy is an obstacle in achieving therapeutic success. The absence of strict correlation between gene mutation class and severity of lung disease suggests a probable role of gene-environmental interactions (such as smoking and virulent bacterial pathogens) or modifier genes besides CFTR that modulate frequency and severity of disease, which warrants further research. ${ }^{5}$ Benefits of ivacaftor therapy cannot be extended to a majority of CF patients who carry at least one copy of the CFTR gene with the $\Delta$ F508 mutation. The issue of optimal duration of ivacaftor therapy will be a key future focus for clinical trials. Long term effects on $\mathrm{FEV}_{1}$, tolerability and survival beyond one year are yet to be disclosed. Safety in infants is another issue to be confirmed in clinical trials. The anticipated cost of therapy is $\$ 294,000$ per year, however a consideration is being proposed for low income group patients. $^{17,19}$

\section{CONCLUSION}

CF is an inherited disorder caused by mutations in the gene encoding CFTR, an ion channel involved in salt and fluid transport in multiple organs. Progressive pulmonary involvement is a major cause of morbidity and mortality. Mutation-class specific pharmacological approaches target at rescuing the poorly biosynthesized or dysfunctional CFTR protein. Ivacaftor is a small molecule drug that facilitates improved chloride transport through the G551D-CFTR channel. In addition to safety, 
significant improvements in biomarkers of CFTR function (NPD and sweat chloride concentration) and lung function have been documented. Ivacaftor represents a significant breakthrough therapy for apparently incurable patients with $\mathrm{CF}$.

\section{REFERENCES}

1. Kabra SK, Kabra M, Lodha R, Shastri S. Cystic fibrosis in India. Pediatr Pulmonol 2007;42:1087-94.

2. Prasad R, Sharma H, Kaur G. Molecular basis of cystic fibrosis disease: an Indian perspective. Indian J Clin Biochem 2010;25:335-41.

3. Ashavaid TF, Raghavan R, Dhairyawan $\mathrm{P}$, Bhawalkar S. Cystic Fibrosis in India: A systematic review. JAPI 2012;60:39-41.

4. Lubamba B, Dhooghe B, Noel S, Leal T. Cystic fibrosis: Insight into CFTR pathophysiology and pharmacotherapy. Clin Biochem 2012;45:1132-44.

5. Boucher RC. Cystic fibrosis. In: Longo DL, Kasper DL Jameson JL, Fauci AS, Hauser SL, Loscalzo J, editors. Harrison's principles of internal medicine. $18^{\text {th }}$ ed. Volume 2. New York: McGraw-Hill; 2012. p. 2147-51.

6. Sloane PA, Rowe SM. Cystic fibrosis transmembrane conductance regulator protein repair as a therapeutic strategy in cystic fibrosis. Curr Opin Pulm Med 2010;16:591-7.

7. Pettit RS. Cystic fibrosis transmembrane conductance regulator- modifying medications: the future of cystic fibrosis treatment. Ann Pharmacother 2012;46:1065-75.

8. Song JC. Ivacaftor: A new emerging treatment option in the management of cystic fibrosis. Formulary 2012;47:132-41.

9. Hussar DA, Eckel SP. Ivacaftor, vismodegib and ingenol mebutate. J Am Pharm Assoc 2012;52:41822.

10. Eckford PDW, Li C, Ramjeesingh M, Bear CE. Cystic Fibrosis Transmembrane Conductance Regulator (CFTR) Potentiator VX-770 (Ivacaftor) Opens the Defective Channel Gate of Mutant CFTR in a Phosphorylation-dependent but ATPindependent Manner. J Biol Chem 2012;287:3663949.
11. Van Goor F, Hadida S, Grootenhuis PD, Burton B, Cao D, Neuberger $\mathrm{T}$, et al. Rescue of CF airway epithelial cell function in vitro by a CFTR potentiator, VX-770. Proc Natl Acad Sci USA 2009;106:18825-30.

12. Accurso FJ, Rowe SM, Clancy JP, Boyle MP, Dunitz JM, Durie PR, et al. Effect of VX-770 in persons with cystic fibrosis and the G551D-CFTR mutation. N Engl J Med 2010;363:1991-2003.

13. Ramsey BW, Davies J, McElvaney NG, Tullis E, Bell SC, Drevinek P, et al. A CFTR potentiator in patients with cystic fibrosis and the G 551D mutation. N Engl J Med 2011;365:1663-72.

14. Aherns R, Rodriguez S, Yen K, Davies JC. VX-770 in subjects 6 to 11 years with cystic fibrosis and the G551D-CFTR mutation (abstract). Pediatr Pulmonol 2011; (suppl 34):283.

15. McKone EF, Borowitz D, Drevinek P, Griese M, Konstan MW, Wainwright CE, et al. Long term safety and efficacy of investigational CFTR potentiator, VX 770, in subjects with CF (abstract). Pediatr Pulmonol 2011; (suppl 34):284.

16. Flume PA, Liou TG, Borowitz DS, Li H, Yen K, Ordonez CL, et al. Ivacaftor in subjects with cystic fibrosis who are homozygous for the F 508 del CFTR mutation (abstract). Pediatr Pulmonol 2011; (suppl 34):284.

17. Buck ML. Ivacaftor for the Treatment of Patients with Cystic Fibrosis and the G551D-CFTR Mutation. Pediatr Pharmacotherapy 2012;18: number 4. Available from http://www.medicine.virginia.edu/clinical/departmen ts/pediatrics/education/pharm-news/2011-

2012/201204.pdf/view. Accessed on 19 September 2012.

18. Yu H, Burton B, Huang CJ, Worley J, Cao D, Johnson JP Jr, et al. Ivacaftor potentiation of multiple CFTR channels with gating mutations. J Cyst Fibros 2012;11:237-45.

19. Sanders DB, Farrell PM. Transformative mutation specific pharmacotherapy for cystic fibrosis. BMJ 2012;344:e79.

doi: 10.5455/2319-2003.ijbcp002912

Cite this article as: Aditya S. Ivacaftor in cystic fibrosis: the first disease modifying agent. Int J Basic Clin Pharmacol 2012;1:225-9. 